\title{
Perceptions and practices for preventing malaria in pregnancy in a peri-urban setting in south-western Uganda
}

\author{
Anthony K. Mbonye ${ }^{1 *}$, Said M. Mohamud² and James Bagonza²
}

\begin{abstract}
Background: Malaria in pregnancy contributes greatly to maternal morbidity and mortality in Uganda. Thus it is urgent to identify possible barriers that limit access to existing interventions. The aim of this study was to assess perceptions and practices regarding malaria prevention during pregnancy in a peri-urban area and explore ways to scale-up malaria prevention interventions, since little is known about malaria in peri-urban settings.

Methods: A survey was conducted in Kabale municipality south-western Uganda from April-June, 2015. Data was collected using a structured questionnaire targeting pregnant women, who delivered in the study area 1 year prior to the survey. Univariate analyses were performed at assess the level of knowledge and practices on malaria prevention during pregnancy.

Results: A total of 800 women was interviewed. The majority of women, $96.1 \% \mathrm{knew}$ that malaria was a dangerous disease in pregnancy; $60.3 \%$ knew that it caused anaemia, and $71.3 \%$ associated malaria with general weakness. However, fewer women (44.9\%) knew that malaria in pregnancy caused abortions, while $14.9 \%$ thought it caused stillbirths. Similarly, few women (19\%) attended the recommend four antenatal care visits; less than a half (48.8\%) accessed two doses of sulfadoxine-pyrimethamine (SP) for malaria prevention in pregnancy while $16.3 \%$ received at least three doses of SP, as recommended by the current policy. The main reasons for poor antenatal care attendance were: women felt healthy and did not see a need to go for antenatal care, long distances and long waiting hours at clinics. The reasons given for not taking SP for malaria prevention were: women were not feeling sick; they were not aware of the benefits of SP in pregnancy, they were sleeping under insecticide-treated nets; fear of side effects of SP; and the antenatal care clinics were far.
\end{abstract}

Conclusion: Despite a good knowledge that malaria is a dangerous disease in pregnancy, there was poor access to antenatal care and use of SP for malaria prevention in pregnancy. There is urgent to address existing health system constraints in order to increases access to malaria prevention in pregnancy in this setting.

Keywords: Malaria in pregnancy, Sulphadoxine-pyrimethamine, Perceptions, Practices, Peri-urban, Uganda

\section{Background}

Uganda has a high burden of malaria in pregnancy that contributes greatly to maternal morbidity and mortality $[1,2]$. The current policy is to give at least three doses of sulfadoxine-pyrimethamine (SP), as intermittent preventive treatment (IPTp) [3]. For treating malaria in

\footnotetext{
*Correspondence: vpadmn@infocom.co.ug; akmbonye@yahoo.com ${ }^{1}$ Ministry of Health, Kampala and School of Public Health-College of Health Sciences, Makerere University, Box 7272, Kampala, Uganda Full list of author information is available at the end of the article
}

pregnancy in first trimester, the policy is to give quinine $10 \mathrm{~m} / \mathrm{kg}$; and artemisinin-based combination therapy (ACT) during the second and third trimester [3].

Interventions to prevent malaria through the public sector have not achieved the desired targets. Recent data shows low utilization of essential health services resulting in missed opportunity. For example, $47.6 \%$ pregnant women attend the required four antenatal care visits; $26.7 \%$ receive the two doses of SP-IPTp while $55.5 \%$ get at least two doses of tetanus toxoid vaccination [4]. The 
previous studies have assessed factors associated with low utilization of health-based interventions, including maternity services and have documented socio-cultural factors, economic, constraints, negative perceptions, poor quality of care and long distances to health units [5-11, 22, 23]. A recent survey in Uganda showed that financial constraints, long distances to the health facilities and concern that drugs were not available were the most significant factors affecting access to health care [4].

There are few epidemiological features of malaria in pregnancy relevant for research as well as deciding on control strategies. First, in malaria endemic areas, the frequency and severity of malaria is greater in pregnant women [12-16]. Second, the severity of clinical manifestations of malaria in pregnancy is determined by the level of pre-pregnancy immunity, which largely depends on the intensity and stability of malaria transmission. Thus, in areas where malaria transmission is low and unstable, the degree of acquired immunity of women is low and both the mother and the foetus may suffer severe consequences of the disease [12]. The third important feature is that in areas of high malaria transmission, primigravidae are more affected [14]. Because of the severe presentation of malaria in low endemic areas, it anticipated that pregnant women in such areas are aware of the severe manifestations and are likely to take preventive actions [17].

Studies on perceptions on malaria in Uganda have shown that malaria is perceived as a common disease $[18,19]$. One study showed that the preventive actions recommended by the women were in line with their perceptions of cause. The use of herbs was mentioned as the first treatment action, followed by the purchase of tablets from shops, with the final recourse being the formal health sector if the previous actions had not led to cure [20]. The aim of this study, therefore, was to assess perceptions and practices on malaria prevention in pregnancy in a pre-urban area in Uganda and explore ways to scale-up these interventions.

\section{Methods Study design}

A survey was conducted in Kabale municipality, southwest Uganda, from April to June, 2015. This area was selected because little is known about malaria in periurban areas. All the three divisions in Kabale municipality participated in the study. A division has a population of approximately 50,000 people and a ward 5000 people. Data collection targeted pregnant who delivered 1 year prior to the survey. The Local Council III offices provided data on divisions, wards and the number of households in each ward. In each ward, a list of households was obtained and all women aged 15-49 years, who had delivered 1 year preceding the survey and consented to participate in the study were interviewed.

\section{Study setting}

The study was conducted in Kabale District, in southwestern Uganda. The district experiences low malaria transmission and occasionally gets malaria outbreaks with severe signs and symptoms of the disease. It is situated $560 \mathrm{~km}$ from the capital city, Kampala and has temperatures ranging from 11 to $24{ }^{\circ} \mathrm{C}$; with a mean annual rainfall of $1000-1250 \mathrm{~mm}$. There are two rainy seasons, the heavy one between March and May, and the lesser one between September and November. Kabale municipality has a total of 32 health facilities including eight government health centres and one referral hospital. All the health facilities offer antenatal care (ANC), distribution of insecticide-treated nets (ITNs), malaria treatment and information on prevention of malaria.

\section{Data sources}

Data was collected on the following variables: sociodemographic characteristics, access to ANC and delivery care, access to malaria prevention services, use of SP for IPTp, and knowledge on adverse effects of malaria and preventions practices. Five research scientists experienced in malaria research conducted the interviews. They underwent refresher training for 3 days on research techniques, and study procedures; and participated in the pretesting and revision of the questionnaire tools before actual field work.

The questionnaire was pretested among 43 participants outside the study area. Some questions on perceptions of malaria in pregnancy that had not been framed well and therefore not understood by the participants were revised for clarity. The final questionnaire was administered in the local language (Rukiga). Said Ishak supervised all aspects of data collection.

\section{Sample size calculation}

Sample size calculation aimed to detect a difference of $5 \%$ in the proportion of women who completed antenatal care visits in the district estimated at $38 \%$ [21]. In order to estimate the proportion with a $\pm 5 \%$ absolute precision, at a power of 80 and $5 \%$ level of significance (two-sided), a minimum sample of 784 pregnant women with $10 \%$ non-participation was targeted for the study.

\section{Data analyses}

Data was entered and verified using Microsoft Access 2007 (Microsoft Inc., Redmond, Washington) and analysed using STATA version 11.0 (STATA Corporation, College Station, Texas). Univariate analyses were performed to calculate proportions on perceptions and preventing practices. Responses from open-ended questions were coded and manually analysed. 


\section{Ethics}

Ethical approval for the research was granted by Makerere University, College of Health Sciences, School of Public health Higher Degrees, Research and Ethics Committee (HDREC). Permission was also granted by the District health office. During field work, an information sheet about the study was given out and written informed consent was sought prior to the interviews.

\section{Results}

A total of 800 women who had delivered a year prior to the survey were interviewed. Their mean age was 29.8 years, range (16-49). The majority were married 701 (87.6 \%) while $328(60.5 \%)$ had attained at least primary education. Women were involved in different activities, 207 (29.9\%) were trading, 281 (35.1 \%) were peasant farmers while 155 (19.4\%) were housewives (Table 1).

The majority of women, 769 (96.1\%) knew that malaria was a dangerous diseases in pregnancy, 469 (58.6\%) knew about HIV/AIDS, 405 (50.6 \%) high blood pressure and 135 (16.9\%) knew about syphilis. Similarly, the majority of women, 482 (60.3 \%) knew that anaemia was due to malaria in pregnancy, 359 (44.9 \%) knew about abortions, 119 (14.9\%) knew about stillbirths and 570 $(71.3 \%)$ associated malaria in pregnancy with general weakness (Table 2).

The majority of women, 451 (83.8 \%) attended at least one ANC visit while few attended the four recommended visits, 102 (19.0\%). The majority received ANC from public facilities, while few, 87 (16.2 \%) received ANC from private facilities (Table 2). ANC attendance

Table 1 Socio-demographic characteristics of respondents

\begin{tabular}{lc}
\hline Socio-demographic characteristics & Frequency (\%) \\
\hline Mean age 29.8 range (16-49 yrs) & \\
Marital status & \\
Married & $701(87.6)$ \\
Divorced & $32(4.0 \%)$ \\
Widowed & $17(2.1 \%)$ \\
Separated & $50(6.3 \%)$ \\
Educational level & \\
No education & $54(6.8 \%)$ \\
Primary & $297(37.1 \%)$ \\
Secondary & $187(23.4 \%)$ \\
Tertiary & $141(17.6 \%)$ \\
University & $120(15.0 \%)$ \\
Occupation & \\
Housewife & $155(19.4 \%)$ \\
Peasant farmer & $281(35.1 \%)$ \\
Trading/business & $207(29.9 \%)$ \\
Employed by the government & $109(13.6 \%)$ \\
\hline
\end{tabular}

was associated with the level of a woman's education, $P=0.0001$; the woman's occupation status, $P=0.001$; partners' education level, $P=0.001$; and the number of children a woman had, $P=0.002$. Results further show that the majority of women, 628 (78.5\%) slept under an ITN a night before the survey; while less than a half, 159 (48.8 \%) accessed two doses of SP. Few, 53 (16.3 \%) received at least three doses of SP as recommended by the current policy (Table 2).

The study solicited reasons why women did not attend ANC and reasons for not taking SP for malaria prevention in pregnancy. The reason given by most women who did not attend ANC was that they were feeling well and they did not see any reason for attending ANC, 213 (81.3); other reasons were: ANC clinics were far, 193 (73.7\%), long waiting hours at clinics, 136 (51.9\%); and husbands did not approve, 42 (16.0\%) (Table 3).

The reason given by most women for not taking SP for malaria prevention in pregnancy was that they were not sick and, therefore, did not see any reason for taking SP, 170 (35.9\%); women were not aware of the benefits of SP in malaria prevention in pregnancy, 85 (18.0\%); and fear of side effects, $38(8.0 \%)$. Others were: the health units were far, 123 (26.0\%); because women were sleeping under ITNs, 80 (16.9\%); and drug stock-outs at health units, 68 (14.4\%) (Table 3).

\section{Discussion}

The present results show that although there was high knowledge that malaria was a dangerous disease in pregnancy, few women knew that malaria in pregnancy caused abortions and stillbirths. Similarly, few women attended the recommend four ANC visits; while few received at least three doses of SP as IPTp as recommended by the current policy. The main reasons for poor ANC attendance were: women felt healthy and did not see a need to go for ANC, long distances to clinics and long waiting hours at clinics; while the reasons given for not taking SP for malaria prevention in pregnancy (IPTp) were: women were not feeling sick; they were not aware of the benefits of SP in pregnancy, fear of side effects of SP, they were sleeping under ITNs; and the ANC clinics were far. Thus there is urgent need to address these constraints in order to increases access to malaria prevention in pregnancy in the study area. Data in Uganda shows that there is relatively high ANC attendance (4 visits) and access to least two doses of tetanus toxoid; yet women who receive two does of SP-IPTp is low calling for interventions to reduce this missed opportunity. This scenario is consistent with findings of a study in subSaharan Africa that show missed opportunities through ANC [22]. Similarly, a previous study has documented constraints to access SP-IPTp such as high user fees, poor 
Table 2 Knowledge and prevention practices on malaria in pregnancy

\begin{tabular}{lc}
\hline Knowledge and prevention practices & $\begin{array}{l}\text { Frequency (\%) } \\
\mathbf{N}=\mathbf{8 0 0}\end{array}$ \\
\hline Knowledge on dangerous diseases in pregnancy & \\
Malaria & $769(96.1 \%)$ \\
HIV/AIDS & $469(58.6 \%)$ \\
Syphilis & $132(16.5 \%)$ \\
High blood pressure & $405(50.6 \%)$ \\
Diabetes & $135(16.9 \%)$ \\
Knowledge on the negative effects of malaria in pregnancy \\
Anaemia & $482(60.3 \%)$ \\
Abortion & $359(44.9 \%)$ \\
Still births & $119(14.9 \%)$ \\
Poor health (general weakness) & $570(71.3 \%)$ \\
Antenatal care attendance & \\
Attended at least once in the last pregnancy & $538(72.5 \%)$ \\
Attended the recommended 4 visits & $102(19.0 \%)$ \\
Attended ANC at public facility & $451(83.8 \%)$ \\
Attended ANC at private facility & $87(16.2 \%)$ \\
Postnatal care attendance & \\
Attended after six weeks of delivery & $473(59.1 \%)$ \\
ITN Use & \\
Slept under and ITN a night before the interview & $628(78.5 \%)$ \\
Taken SP for malaria prevention & \\
Taken SP & $326(40.8 \%)$ \\
Taken the two recommended doses of SP & $159(48.8 \%)$ \\
Taken three doses of SP & $53(16.3 \%)$ \\
Taken four doses of SP & $15.6 \%)$ \\
\hline & \\
&
\end{tabular}

Table 3 Reasons for not attending ANC and not taking SP as IPTp

\begin{tabular}{ll}
\hline & Frequency (\%) \\
\hline Reason for not attending ANC & $\mathrm{N}=262$ \\
Was feeling healthy & $213(81.3 \%)$ \\
Was busy & $52(19.9 \%)$ \\
ANC clinic was too far & $193(73.7 \%)$ \\
Long waiting hours at the clinic & $136(51.9 \%)$ \\
Husband did not allow & $42(16.0 \%)$ \\
There is poor quality at the clinic & $27(10.3 \%)$ \\
Reasons for not taking SP for malaria prevention & $\mathrm{N}=473$ \\
Because I was sleeping under an ITN & $80(16.9 \%)$ \\
I was not sick & $170(35.9 \%)$ \\
The health unit was far & $123(26.0 \%)$ \\
There were no drugs at the ANC clinic & $68(14.4 \%)$ \\
I was not aware of the use of SP & $85(18.0 \%)$ \\
I did not have money & $65(13.7 \%)$ \\
The health worker was busy & $43(9.1 \%)$ \\
I feared side effects & $38(8.0 \%)$ \\
I used herbs & $25(5.3 \%)$ \\
\hline
\end{tabular}

quality of care, frequent drug stock-outs and confusing polices and guidelines on SP-IPTp [23].

The present findings highlight the importance of how the awareness about a disease and the way people perceive themselves at risk of that disease influences the preventive actions taken as previously described in the Health Belief Model [17]. As shown, although women knew that malaria was a dangerous disease in pregnancy and caused anaemia, few associated it with loss of pregnancy through abortions and stillbirths. It is possible this perception contributed to few women taking SP for malaria prevention in pregnancy. To compound this, few women knew the benefits of SP and they feared side effects of SP.

The present results are consistent with findings of an earlier study in the same district which showed that ANC attendance was irregular and few women knew that the purpose of attending antenatal care was to monitor both the growth of the baby and the health status of the woman. Health-seeking behaviour was influenced by several factors, including the perceived high cost of ANC services, and perceived inadequacy of services provided by the formal health system. Inadequacy of formal health services was perceived by users to be partly due to understaffing and to irregular supply of essential drugs [20].

The present results are also consistent with findings of another study carried out in a high malaria endemic and largely a rural district in Uganda which showed that that primigravidae, adolescents and men were not considered at risk of malaria. Similarly anaemia and low birth weight were not associated with malaria; and most women could not differentiate symptoms of malaria from those of early pregnancy [19].

While interpreting these results there is need to be cautious about several limitations. This study was carried out in a mainly urban area and may not represent rural women who may have difficulties in accessing information and health services. Thus the perceptions and health-seeking practices could be different. Similarly, this was a rapid survey and did not explore fully perceptions and health-seeking behaviour related to malaria in pregnancy and this is recommended this for further studies.

The study in a peri-urban area is timely since little is known about malaria prevention practices in such settings. Yet with increasing urbanisation in Uganda, there is need to identify barriers that could help in designing delivery of malaria prevention interventions. It is shown that a high proportion of the study population had attained secondary education thus could appreciate health messages and the benefits of malaria prevention in pregnancy and possibly adopting positive behaviour practices. Since there has been wide distribution and use of ITNs in Uganda; (78.5 \% in the study area); there 
is need for further studies to explore how the changing malaria endemicity will affect perceptions and practices in such settings.

The immediate policy implication of these results is create a health promotion package that explains the benefits of SP for malaria prevention in pregnancy, its dangers and the importance of attending ANC to receive treatment and prevention services; as previously recommended [24]. However, there was a substantial number $(16 \%)$ of women in the present study who were not allowed to attend ANC by their husbands. Thus engaging men in promoting maternal health should be part of this package.

These results will be discussed with the district health team to enable them design this package. The medium term policy implications is to discuss health system strengthening interventions to ensure improved quality of care and modalities of bring services close to pregnant women through regular outreach programmes.

In conclusion, these results show that there is still a disconnect between what women know about malaria, its negative effects and health-seeking behaviour. To increases access to malaria prevention in pregnancy, there is need to create both awareness on malaria in pregnancy as well strengthening the health system to provide quality and acceptable services in this district.

\section{Authors' contributions}

AKM conceived the study. SM supervised data collection and JB supervised data collection and critically reviewed the manuscript. AKM, SM and JB participated in the design of the study. AKM wrote the first draft of the manuscript. All authors have read and approved the final manuscript.

\section{Author details}

${ }^{1}$ Ministry of Health, Kampala and School of Public Health-College of Health Sciences, Makerere University, Box 7272, Kampala, Uganda. ${ }^{2}$ School of Public Health, College of Health Sciences, Makerere University, Kampala, Uganda.

\section{Acknowledgements}

We are grateful to the research assistants who collected the data; the district health officer Kabale, Dr. Patrick Tusiime, the local council leaders and all the women who participated in this study.

The study was supported by the Ministry of Health, Directorate of Clinical and Community Health Services, Ministry of Health, Uganda.

\section{Competing interests}

The authors declare that they have no competing interests.

Received: 22 November 2015 Accepted: 30 March 2016 Published online: 14 April 2016

\section{References}

1. Mbonye AK, Bygbjerg I, Magnussen P. Intermittent preventive treatment of malaria in pregnancy: a community-based delivery system and its effect on parasitaemia, anemia and low birth weight in Uganda. Int J Infect Dis. 2008;12:22-9.
2. Mbonye AK, Bygbjerg IC, Magnussen P. Intermittent preventive treatment of malaria in pregnancy: a new delivery system and its effect on maternal health and pregnancy outcomes in Uganda. Bull World Health Organ. 2008;86:93-100.

3. Ministry of Health. Uganda Clinical Guidelines. Ministry of Health, Box 7272, Kampala, Uganda, 2012.

4. Uganda Bureaux of Statistics. Uganda Demographic and Health Survey. Kampala: Uganda Bureaux of Statistics; 2011.

5. Mbonye AK, Neema S, Magnussen P. Malaria in pregnancy, risk perceptions and care seeking practices among adolescents in Mukono district Uganda. Int J Adolesc Med Health. 2006;18:561-73.

6. Mbonye AK. Prevalence of childhood illnesses and care-seeking practices in rural Uganda. Sci World J. 2003;3:721-3.

7. Mbonye AK, Sentongo M, Mukasa GK, Byaruhanga R, Sentumbwe-Mugisa O, Waiswa P, et al. Newborn survival in Uganda: a decade of change and future implications. Health Policy Plan. 2012;27(Suppl 3):104-17.

8. Ocan M, Bwanga F, Bbosa GS, Bagenda D, Waako P, Ogwal-Okeng J, et al. Patterns and predictors of self-medication in northern Uganda. PLoS One. 2014;9:e92323.

9. Heggenhougen HK, Hackenthal V, Vivek P. Bed nets usage and its acceptance at the local level. In: The behaviour and social aspects of malaria control: an introduction and annotated biography. Special programme for research and training in tropical diseases (TDR); 2003. pp. 97-106.

10. Helitzer-Allen DL, McFarland DA, Wirima JJ, Macheso AP. Malaria chemoprophylaxis compliance in pregnant women: a cost-effectiveness analysis of alternate interventions. Soc Sci Med. 1993;36:403-7.

11. Mbonye AK, Magnussen P, Bygbjerg IB. Intermittent preventive treatment of malaria in pregnancy: the effect of new delivery approaches on access and compliance rates in Uganda. Trop Med Int Health. 2007;12:519-31.

12. Menendez C. Malaria in pregnancy: a priority area of malaria research and control. Parasitol Today. 1995;11:178-83.

13. Brabin B. An analysis of malaria in pregnancy in Africa. Bull World Health Organ. 1983;61:1005-16.

14. McGregor IA, Wilson ME, Bilewicz WZ. Malaria infection of the placenta in The Gambia, West Africa, its incidence and relationship to birth weight and placenta weight. Trans R Soc Trop Med Hyg. 1983;93:529-34.

15. Greenwood B. The use of anti-malarial drugs to prevent malaria in the population of malaria-endemic areas. Am J Trop Med Hyg. 2004;70:1-7.

16. Guyatt HL, Noor AM, Ochola SA, Snow RW. Use of intermittent presumptive treatment and insecticide-treated bed nets by pregnant women in four Kenyan districts. Trop Med Int Health. 2004;9:255-61.

17. Becker MH. The health belief model and personal health behaviour. Health Education Monographs. 1974;2:4.

18. Kengeya-Kayondo JF, Seeley JA, Kajura-Bajenja E, Kabunga E, Mubiru E, Sembajja F, et al. Recognition, treatment seeking behaviour and perception of cause of malaria among rural women in Uganda. Acta Trop. 1994;58:267-73.

19. Mbonye AK, Neema S, Magnussen P. Treatment-seeking practices for malaria in pregnancy among rural women in Mukono district, Uganda. J Biosoc Sci. 2006;38:221-37.

20. Ndyomugyenyi R, Neema S, Magnussen P. The use of formal and informal services for antenatal care and malaria treatment in rural Uganda. Health Policy Plan. 1998;13:94-102.

21. Ministry of Health. Annual Report. Ministry of Health, Box 7272, Kampala, Uganda, 2014

22. Andrews KG, Lynch M, Eckert E, Gutman J. Missed opportunities to deliver intermittent preventive treatment for malaria to pregnant women 2003-2013: a systematic analysis of 58 household surveys in sub-Saharan Africa. Malar J. 2015;14:521.

23. Hill J, Hoyt J, van Eijk AM, D'Mello-Guyett L, Ter Kuile FO, Steketee R, et al. Factors affecting the delivery, access, and use of interventions to prevent malaria in pregnancy in sub-Saharan Africa: a systematic review and meta-analysis. PLoS Med. 2013;10:e1001488.

24. Chico RM, Dellicour S, Roman E, Mangiaterra V, Coleman J, Menendez C, et al. Global Call to Action: maximize the public health impact of intermittent preventive treatment of malaria in pregnancy in sub-Saharan Africa. Malar J. 2015;14:207. doi:10.1186/s12936-015-0728-x. 